\title{
A Wireless Micro Inertial Measurement Unit (IMU)
}

\author{
F. Höflinger ${ }^{1}$, J. Müller ${ }^{2}$, M. Törk ${ }^{1}$, L.M. Reindl ${ }^{1}$, W. Burgard ${ }^{2}$
}

${ }^{1}$ IMTEK, University of Freiburg, Germany, \{fabian.hoeflinger, maximilian.toerk, reindl $\} @$ imtek.uni-freiburg.de ${ }^{2}$ Department of Computer Science, University of Freiburg, Germany, \{muellerj, burgard\}@informatik.uni-freiburg.de

\begin{abstract}
In this paper, we present a wireless micro inertial measurement unit (IMU) with the smallest volume and weight requirements available at the moment. With a size of $18 \mathrm{~mm} \times$ $16 \mathrm{~mm} \times 4 \mathrm{~mm}$, this IMU provides full control over the data of a three-axis accelerometer, a three-axis gyroscope, and a threeaxis magnetometer. It meets the design prerequisites of a spacesaving design and eliminates the need of a hard-wired data communication while still being comparable to state of the art commercially available MEMS IMUs. A CC430 microcontroller sends the collected raw data to a base station wirelessly with a maximum sensor sample rate of 640 samples per second. Thereby, the IMU performance is optimized by moving data post processing to the base station. This development offers important features in embedded microsystem applications with their significant size and weight requirements.
\end{abstract}

Index Terms-Inertial Measurement Unit, Wireless Sensor, Allan Variance

\section{INTRODUCTION}

In recent years, location-based services for indoor mobile devices are becoming more and more popular due to their increasing functional range. In the context of indoor applications, where GPS signals are not available, one promising localization approach, e.g., for cell phones, people, or mobile robots, relies on inertial sensors. For human tracking, miniature inertial sensors integrated into clothes or shoes can extend the functionality of pedometers by full 3D location information. Even more complex tasks like full-body human motion capture have been addressed using inertial sensors. Additionally, the combination of inertial sensors with wireless signal sensors allows for correction of the errors accumulated during integration of inertial sensor data as shown by Xsens with the MVN MotionGrid. Similarly, personal devices like cell phones can be localized inside of buildings. In the domain of mobile robots inertial sensors are commonly used in combination with cameras for flight stabilization or autonomous hovering of helicopters or quadrotors. Furthermore, inertial sensors have been applied for localization of airplanes and miniature indoor blimps.

For application in such devices or on robots, small commercially available inertial measurement units (IMUs) typically incorporate MEMS acceleration sensors, gyroscopes, and magnetometers measuring in three axis. The Sparkfun Electronics Razor IMU [1] combines those sensors with an AT328 microcontroller and provides access to the raw sensor data. Additionally, most of the commercially available IMUs like the Xsens MTi [2] offer a measurement data preprocessing and robust on-board sensor data fusion for 3D orientation. However, due to their size, weight, or power consumption, these IMUs are not ideally suitable for certain applications such as miniature aerial vehicles [3], [4], [5] or inconspicuous integration into clothes or shoes.

Apart from commercial products, several research groups have put effort into enhancing IMUs concerning special characteristics. One important aim, for example, is the miniaturization of the device. Barton et al. demonstrated a cubic IMU design with a side length of $10 \mathrm{~mm}$ and wireless communication [6]. However, they employ analog sensors which demand for separate analog-to-digital converters and a precise reference voltage. Lin et al. presented a system for the analysis of yaw movements with dimensions of $37 \mathrm{~mm} \times 23 \mathrm{~mm} \times 12 \mathrm{~mm}$, integrated with a bluetooth module and a lithium polymer battery which is comparably large and has limited functionality [7]. Another approach is the combination of typical accelerometers and gyroscopes with a linear encoder for which Lim et al. claim a size of $10 \mathrm{~mm} \times 15 \mathrm{~mm}$ [8]. Depending on their application Barton et al. change the priorities to maximized data throughput as demonstrated in [9].

\section{Design OF THE MiCRo-IMU}

To date the commercially available IMUs are not implementable in small mobile objects due to the constraints like big size, high power consumption and portability issues. In contrast to the commercial products, existing research prototypes are smaller and lighter but the data performance is a bottle neck. For the data processing it is important to have high raw data rates.

In our methodology of designing the Micro-IMU, we combined the aim of an applicable IMU for embedded microsystems and improved the characteristics in size, weight, and power consumption while the performance is still comparable to state-of-the-art commercially available MEMS IMUs. By using MEMS sensors with large scale integration this becomes possible. Modern accelerometers and gyroscopes feature three-axis technology and integrated analog-to-digital conversion with automatic temperature compensation in a onechip-design. This saves space in the IMU design as analog converters, high precision voltage references and 3D packaging are not necessary. Thus, a four-layer PCB is sufficient for integration of all sensors and a microcontroller.

One important aspect of the design is the decision to move data-processing from the IMU to a base station. Through this 


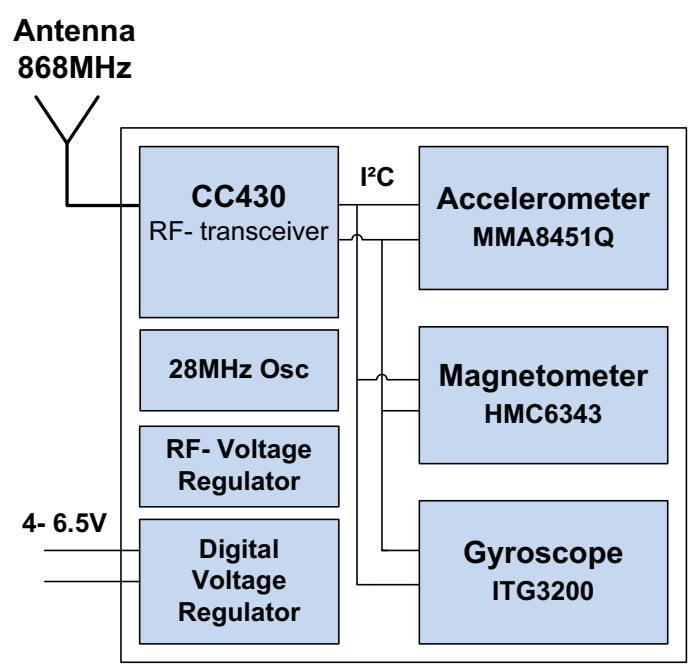

Fig. 1. Block diagram of the Micro-IMU

TABLE I

SPECIFICATION OF THE SENSORS

\begin{tabular}{|c|c|c|c|c|}
\hline $\begin{array}{l}\text { Sensor } \\
\text { Type }\end{array}$ & $\begin{array}{l}\text { Full-Scale } \\
\text { Range }\end{array}$ & Sensitivity & $\begin{array}{l}\text { Sampling } \\
\text { Rate }\end{array}$ & $\begin{array}{l}\text { Digital } \\
\text { Resolution }\end{array}$ \\
\hline $\begin{array}{l}\text { Accelerometer } \\
\text { MMA8451Q } \\
\text { Gyroscope }\end{array}$ & $\pm 4 \mathrm{~g}$ & $0.003 \mathrm{~g}$ & 800 samples/s & 14 bit \\
\hline $\begin{array}{l}\text { ITG3200 } \\
\text { Magnetometer }\end{array}$ & $2000^{\circ} / \mathrm{s}$ & $0.061^{\circ} / \mathrm{s}$ & $8 \mathrm{k}$ samples/s & 16 bit \\
\hline HМC6343 & $\pm 1 \mathrm{Gs}$ & $0.1^{\circ}$ & 10 samples/s & 12 bit \\
\hline
\end{tabular}

step, the power consumption of the IMU can be reduced, as the demanding computation of sensor data fusion filters and other algorithms to enhance the data quality is moved to more powerful computers with less constraints in size and weight. In this case the energy source of the IMU can be minimized in weight and size or the lifetime can be increased, respectively. Especially for battery powered embedded microsystems, this is important.

The block components of the designed Micro-IMU are shown in Fig. 1. The CC430 microcontroller is interfaced to the 3-Axis accelerometer (MMA8451Q), 3-Axis magnetometer (HMC6343), and 3-Axis gyroscope (ITG3200) via the $\mathrm{I}^{2} \mathrm{C}$ protocol. A $28 \mathrm{MHz}$ oscillator is used for the transceiver functionality along with a voltage regulator for the RFcircuitry. Furthermore, a separated voltage regulator for the digital circuitry is used to avoid high frequency distortions in the sensor data.

Table I shows the specification of the sensors implemented on the Micro-IMU. The sensors are systematically selected for a high data rate and best digital resolution while providing full scale range at the same time.

For reading the digital sensor data and radio data transmission a CC430 microcontroller is used, which combines an integrated CC1101 wireless transceiver and an MSP430 microcontroller. The MSP430 is a 16-bit RISC mixed-signal processor for ultra low power applications.

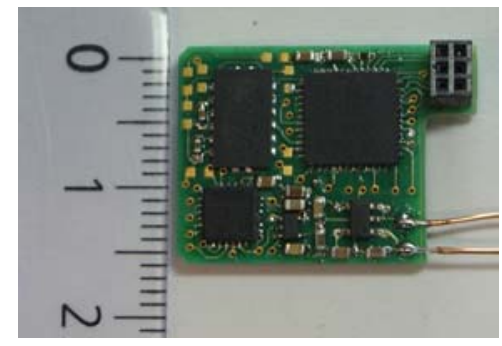

Fig. 2. Micro-IMU top view

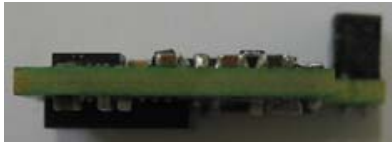

Fig. 3. Micro-IMU side view

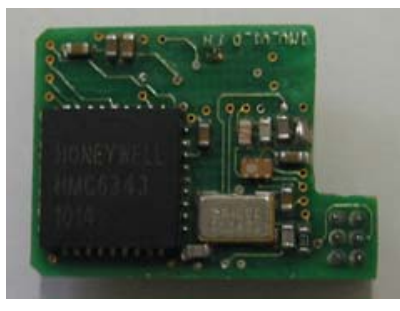

Fig. 4. Micro-IMU bottom view
The wireless transceiver works in the SRD-Band (a band designed for low-power communication devices), which is specified in the European Union from $863-870 \mathrm{MHz}$ with a maximum allowed power output of $10 \mathrm{dBm}$. The low-power communications protocol SimpliciTI from Texas Instruments is used, as this protocol is efficient due to the minimal frame overhead. The transceiver part of the CC430 supports the maximum allowed power output i.e. $10 \mathrm{dBm}$. Further using CC430 a maximum radio transmission data rate of $500 \mathrm{kbaud} / \mathrm{sec}$ is achieved, whereby the maximum distance is depending on the data rate, hence decreasing the receiver sensitivity. For validation of the communication link a hardware CRC16 module is implemented on the CC430 which has a performance advantage for the microcontroller. Consequently the sole purpose of the CC430 microcontroller is to collect the raw sensor data at the maximum sensor data rate and transmit it to the host with minimal power consumption. This is accomplished with a maximum sensor sample rate of 640 samples per second.

By choosing QFN-packages and using a four-layer PCB layout the size of the IMU is reduced almost to the accumulated footprint of the ICs and measures $18 \mathrm{~mm} \times 16 \mathrm{~mm} \times 4 \mathrm{~mm}$. The two inner layers are floated with ground copper to have a large ground plane for the quarter-wave antenna. The ground plane acts as the "missing half" of a dipole antenna. The large size of ground plane is important for the radiation quality of the antenna. Furthermore, using an increased ground plane reduced the noise floor and minimzes the overall resistance as all devices are connected to this ground plane. SWRA250 from Johanson Technology is used to provide on-chip impedance matching between the transceiver and antenna of the designed Micro-IMU. Subsequently this single chip, i.e. SWRA250, reduces the size of matching network which could have been worse if a procedural impedance matching network was used. A picture of the top-view is given in Fig. 2. Instead of a 


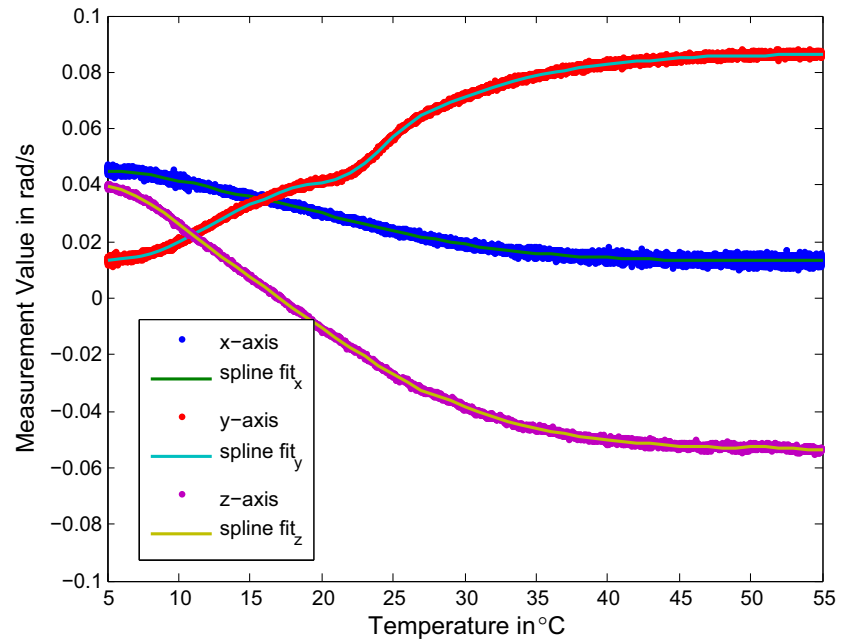

Fig. 5. Gyroscope ITG3200 drift

chip antenna, we employ a quarter-wave wire-antenna on the Micro-IMU which can be adjusted to the available space on the embedded system. Fig. 3 shows the side view dimensions of our Micro-IMU. The programming port on the top right is optional and can be removed for further size reduction. The high frequency components are implemented in the bottom layer of the designed PCB (shown in Fig 4) in order to avoid high frequency distortions.

For reception of the raw IMU sensor data we developed a hardware module which can be connected to a standard PC via USB. This hardware module applies a CRC check to the incoming data stream and automatically deletes invalid packets.

\section{DATA PROCESSING}

One important factor concerning the quality of the position and orientation estimates of the IMU is the data postprocessing. In this paper, we present techniques for data refinement and characterization of deviations, examining the static case of immobility.

\section{A. Compensation}

Some systematic deviations, namely offset, temperature drift, and cross-axis effect, on the sensor readings can be compensated by calibration.

For offset calibration the average output value of each sensor over a time span of $5 \mathrm{~min}$ is determined in the rest position. This value is then subtracted from the subsequent sensor output.

To analyze the temperature drift of the gyroscope and the accelerometer, we expose the IMU to temperature curves in a climate chamber from $5^{\circ} \mathrm{C}$ to $55^{\circ} \mathrm{C}$. Although the implemented digital gyroscope has a built in temperature compensation, it still exhibits a distinct temperature drift, which is shown in Fig. 5. Additionally the noise of the sensor is increasing with the value of temperature. This effect is caused by the electronic noise which is generated by the thermal agitation of the charge carriers inside a semiconductor.
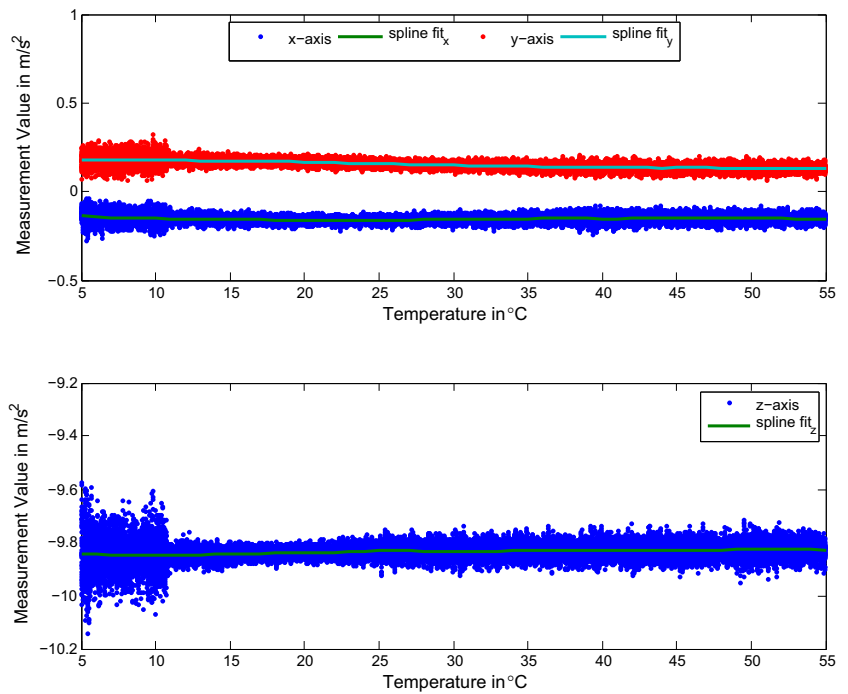

Fig. 6. Accelerometer MMA8451Q drift

The CC430 microcontroller has an internal temperature diode, which can be used to measure the temperature through the internal analog digital converter. The temperature sensor has a linear output over the temperature range from $-10^{\circ} \mathrm{C}$ to $85^{\circ} \mathrm{C}$. However, its offset error can be large and needs to be calibrated precisely. The temperature data is transmitted wirelessly together with the sensor data to the base station. For temperature drift compensation, we applied a least-squares cubic spline approximation to the data recorded in the climate chamber as shown in Fig. 5.

The digital accelerometer has no significant temperature drift, which is shown in Fig. 6. The increased noise in the first part of the measured sensor curve is caused by vibrations of the heating mechanics of the climate chamber.

Concerning the magnetic field sensors, the cross-axis effect has to be taken into account [10]. This effect causes a slight sensitivity to a magnetic field orthogonal to the actual measuring axis. If the actual magnetic field is $m=\left[m_{x}, m_{y}, m_{z}\right]^{T}$ the sensors measure a field $m^{\prime}=\left[m_{x}^{\prime}, m_{y}^{\prime}, m_{z}^{\prime}\right]^{T}$ with

$$
m^{\prime}=C m+m_{0}
$$

where the matrix $C$ includes the cross-axis effect and $m_{0}$ denotes the zero offset. From the measured values $m^{\prime}$ the real magnetic field can be calculated as $m=K m^{\prime}+m_{0}^{\prime}$ using the calibration matrix $K=C^{-1}$ and the shift $m_{0}^{\prime}=C^{-1} m_{0}$ by

$$
m=K m^{\prime}+m_{0}^{\prime}
$$

To measure these cross-sensitivities we mount the IMU on a robot and rotate it around all three axes thereby measuring the geomagnetic field. We then use the standard deviation of the measurement in the rotation axis and the mean of the measurements of the not rotating axes as an evaluation criterion of the parameters $K$ and $m_{0}^{\prime}$. For achieving the same scale on each axis, the deviation of the measurement along the rotation axis from the expected measurement value is chosen as an additional criterion. With these criteria a randomized 


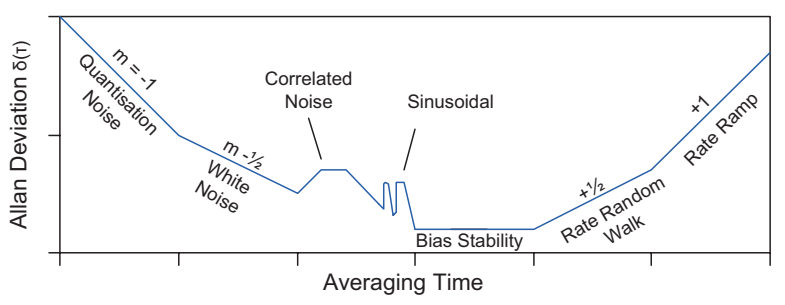

Fig. 7. Schematic diagram of the Allan variance

search is performed in the parameter space and the best fitting parameters are used as a starting point in a gradient decent optimization.

\section{B. Permanent Measurement Error and Allan Variance}

After compensation of static measurement deviations, the measurement data quality is determined by bias-drift and noise. The drift of the measurement values of the gyroscopes and accelerometers has a devastating effect on the on accuracy of an IMU. This drift introduces a small changing bias offsets which corrupts the data processed by the IMU. As a consequence, this error leads to a quadratic effect on the position estimated by integration of the measurement data. To gain knowledge about the magnitude of drift and noise we evaluate the Allan variance $\sigma_{a}^{2}$ of the measurement data. The graph of the Allan variance expresses five types of noise terms with various gradients as shown in Fig. 7. The noise is identified by measuring the slope of the curve at the averaging time of $1 \mathrm{~s}$. The drift is defined as the y-coordinate at the minimum of the Allan variance.

The Allan variance is an instrument for representing the root mean square random drift errors as a function of averaging time intervals $\Delta t$ [11]. It is defined as

$$
\sigma_{a}^{2}(\Delta t)=\frac{1}{2(N-1)} \sum_{i=1}^{N-1}\left(x_{i}-x_{i-1}\right)^{2}
$$

depending on the differences between consecutive measurements of the sensor data recorded at the measurement period $\Delta t$.

\section{Results}

For the sensor characterization by means of of the Allan variance, at first we captured the raw sensor data of the IMU with a the maximum sample rate of 340 samples per second for four hours. The resulting curve for the gyroscope of the MicroIMU is depicted in Fig. 8 and the precise characterization values for noise and drift are presented in Table III. Comparing the three axes, the $\mathrm{z}$-axis shows a minimum that is significantly lower than the other two. This effect can be explained by the MEMS-nature and the geometry of the sensor. The structures for all three axes are manufactured in one planar chip design. Therefore, trade-offs have to be made concerning the sensor quality in certain measurement axes.

Regarding the acceleration sensor shown in Fig. 9 a similar distinction between the z-axis and the other two axis can be

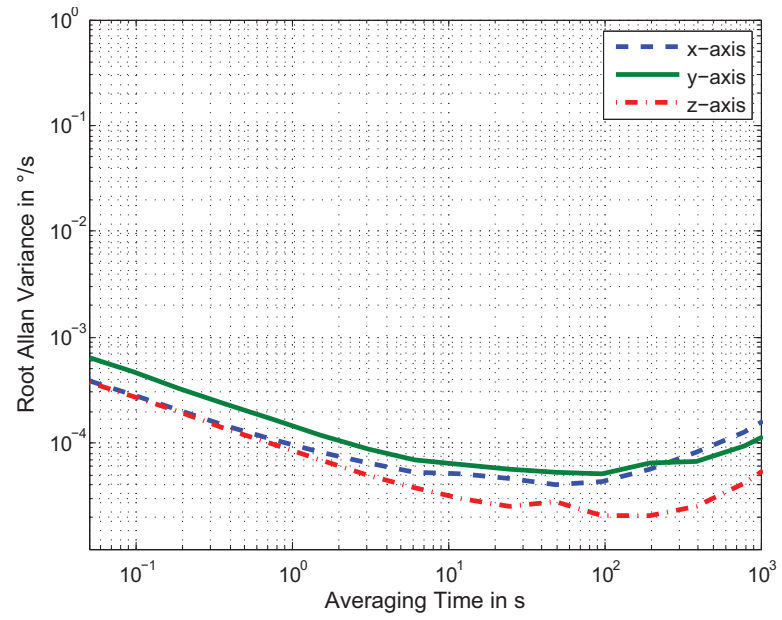

Fig. 8. Allan variance of the gyroscope ITG3200 of the Micro-IMU

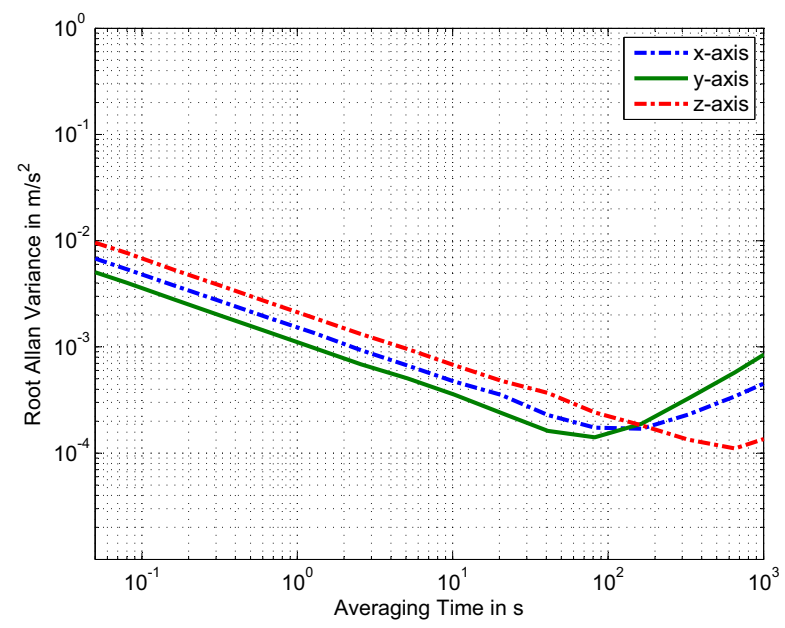

Fig. 9. Allan variance of the acceleration sensor MMA8451Q of the MicroIMU

observed. Again the MEMS-nature causes certain priorityorientations, in this case the quality of the noise in the $\mathrm{x}$ and $y$-axis is improved. The drift of the sensor is similar in all three axis. The exact characterization values are given in Table III.

In our first prototype of the Micro-IMU we used the STMicroelectronics LIS3LV02 acceleration sensor. The Allan Variance of this sensor is shown in Fig. 10. The values of the noise and drift factor are given in Table II. As shown in Fig. 10 the noise and drift factor on $\mathrm{z}$-axis is ten times worse compared with the other two axis. Consequently, we replaced the LIS3LV02 sensor by the MMA8451Q sensor which is utilized in our latest prototype of the Micro-IMU.

\section{Comparison with Xsens}

In order to compare the Micro-IMU with the Xsens MTi [2], we collected a set of raw data from the Xsens MTi over a period of four hours with a data rate of 100 samples per second. We analyzed this data with help of the Allan variance. 


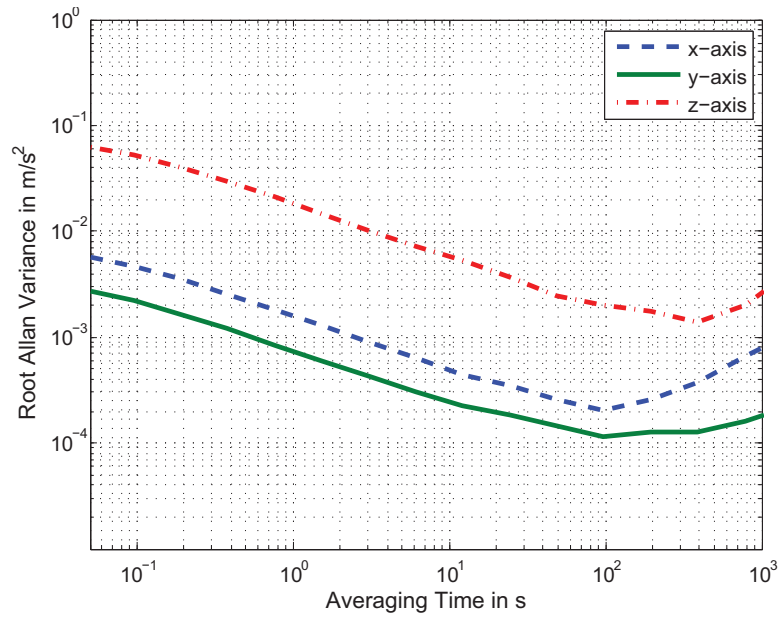

Fig. 10. Allan variance of the acceleration sensor LIS3LV02

TABLE II

DATATSHEET OF ACCELERATION SENSOR LIS3LV02

\begin{tabular}{ll}
\hline Acceleration sensor LIS3LV02 \\
\hline Noise in $m / s^{2} / \sqrt{H z}$ & \\
X-axis & 0.00150 \\
y-axis & 0.00072 \\
Z-axis & 0.01700 \\
Drift in $m / s^{2}$ & \\
X-axis & 0.00021 \\
y-axis & 0.00012 \\
Z-axis & 0.00140 \\
\hline
\end{tabular}

The obtained curves are shown in Fig. 11 and 12 and the precise characteristics can be found in Table III.

Comparing the IMUs the most distinctive difference is the better homogeneity of the Xsens MTi concerning the axes. This is due to the fact that for each axis a single integrated sensor is used. The Xsens MTi uses a 3D PCB-design witch allows to place each sensor on one of the three orthogonally placed PCBs.

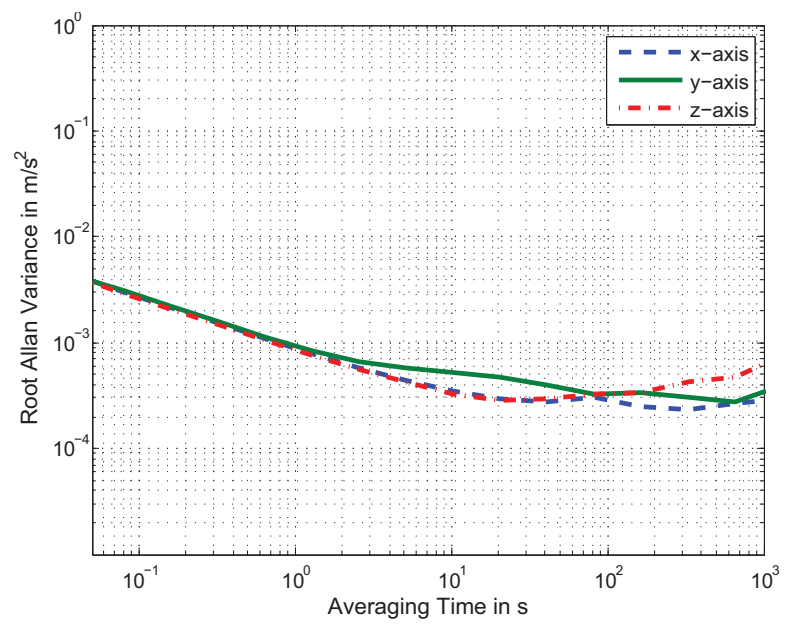

Fig. 11. Allan variance of the acceleration sensor of the Xsens MTi

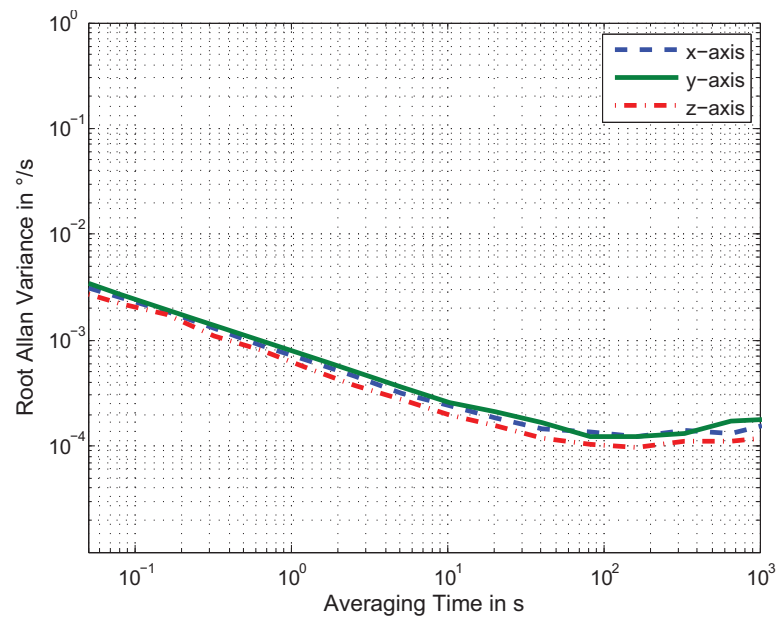

Fig. 12. Allan variance of the gyroscope of the Xsens MTi

TABLE III

COMPARISON BETWEEN XSENS [2] AND MICRO-IMU

\begin{tabular}{|c|c|c|}
\hline & Micro-IMU & Xsens MTi \\
\hline Interface & Wireless & RS232/USB \\
\hline Transmitting Power & $10 \mathrm{dBm}$ & (wired) \\
\hline Transmission Distance & $10 \mathrm{~m}$ & (wired) \\
\hline Transfer Rate & 250 kbaud & 961 kbaud \\
\hline Sampling Rate & 640 sample/sec & 100 sample/sec \\
\hline Power Consumption & $110 \mathrm{~mW}$ & $350 \mathrm{~mW}$ \\
\hline Supply Voltage & $3.6-6 \mathrm{~V}$ & $4.5-30 \mathrm{~V}$ \\
\hline Price & $€ 150$ Production & $€ 2500$ \\
\hline Size (OEM) & $18 \times 16 \times 4 \mathrm{~mm}^{3}$ & $48 \times 33 \times 15 \mathrm{~mm}^{3}$ \\
\hline Weight (OEM) & $3 \mathrm{~g}$ & $11 \mathrm{~g}$ \\
\hline \multicolumn{3}{|l|}{ Gyroscope } \\
\hline \multicolumn{3}{|l|}{ Noise in ${ }^{\circ} / s / \sqrt{H z}$} \\
\hline $\mathrm{x}$-axis & 0.0055 & 0.045 \\
\hline$y$-axis & 0.0082 & 0.041 \\
\hline $\mathrm{z}$-axis & 0.0049 & 0.036 \\
\hline \multicolumn{3}{|l|}{ Drift in $\% / s$} \\
\hline $\mathrm{x}$-axis & 0.0023 & 0.0070 \\
\hline$y$-axis & 0.0030 & 0.0069 \\
\hline $\mathrm{z}$-axis & 0.0012 & 0.0055 \\
\hline \multicolumn{3}{|l|}{ Acceleration Sensor } \\
\hline \multicolumn{3}{|l|}{ Noise in $m / s^{2} / \sqrt{H z}$} \\
\hline $\mathrm{x}$-axis & 0.00150 & 0.00090 \\
\hline$y$-axis & 0.00110 & 0.00095 \\
\hline $\mathrm{z}$-axis & 0.00205 & 0.00085 \\
\hline \multicolumn{3}{|l|}{ Drift in $m / s^{2}$} \\
\hline $\mathrm{x}$-axis & 0.00017 & 0.00023 \\
\hline$y$-axis & 0.00014 & 0.00027 \\
\hline $\mathrm{z}$-axis & 0.00016 & 0.00029 \\
\hline
\end{tabular}

Looking at the data of the accelerometer the noise is in the same range, except for the z-axis where the MTi performs significantly better than the Micro-IMU. The drift are in average two times higher for the MTi than for the Micro-IMU.

For the gyroscope the Micro-IMU outperforms the MTi concerning drift and noise. The noise level of the Micro-IMU is in average six times lower than that of the MTi and the drift is lower by a factor of three.

Overall, despite of its small size, the performance of our Micro-IMU showed to be comparable to that of the Xsens MTi in an extensive static evaluation. 


\section{CONClusion AND Future Work}

In this paper, we presented the design of a wireless, lowpower IMU that features minimum size and weight. By using highly integrated digital sensors and relocating data postprocessing from the IMU to a base station, we are capable of competing with state-of-the-art commercial MEMS IMUs such as the Xsens MTi. Due to its favorable properties, our Micro-IMU can be applied for tracking of miniature moving objects or small-size autonomous robots especially with limited payload and energy.

In our further development we will additionally characterize our Micro-IMU in the non-static case. Besides the evaluation of the linearity of the sensors we would like to evaluate the dynamic measurement noise of the Micro-IMU in its target application. For that, we plan to utilize an optical motion capture system providing high-precision position references.

\section{ACKNOWLEDGMENT}

This work has partly been supported by the German Research Foundation (DFG) within the Research Training Group 1103 (Embedded Microsystems) and by the Gottfried Wilhelm Leibniz Program of the DFG.

\section{REFERENCES}

[1] "Sparkfun Razor IMU," 2011, [accessed 2011/11/04]. [Online]. Available: http://www.sparkfun.com/products/10736

[2] "Xsens MTi," 2011, [accessed 2011/11/04]. [Online]. Available: http://www.xsens.com/en/general/mti
[3] J. Müller, A. Rottmann, L. Reindl, and W. Burgard, "A probabilistic sonar sensor model for robust localization of a small-size blimp in indoor environments using a particle filter," in Proc. of the IEEE Int. Conf. on Robotics \& Automation (ICRA), 2009

[4] D. Schafroth, S. Bouabdallah, C. Bermes, and R. Siegwart, "From the test benches to the first prototype of the muFly micro helicopter," Journal of Intelligent \& Robotic Systems, vol. 54, no. 1-3, 2009.

[5] R. Woods, S. Avadhanula, E. Steltz, M. Seeman, J. Entwistle, A. Bachrach, G. Barrows, S. Sanders, and R. Fearing, "An autonomous palm-sized gliding micro air vehicle," IEEE Robotics \& Automation Magazine, vol. 14, no. 2, 2007.

[6] J. Barton, A. Gonzalez, J. Buckley, B. O'Flynn, and S. O'Mathuna, "Design, fabrication and testing of miniaturised wireless inertial measurement units (IMU)," in Electronic Components and Technology Conference, 2007. ECTC '07. Proceedings. 57th, 29 2007-june 12007 , pp. $1143-1148$.

[7] Z. Lin, M. Zecca, S. Sessa, L. Bartolomeo, H. Ishii, K. Itoh, and A. Takanishi, "Development of the miniaturized wireless inertial measurement unit wb-4: Pilot test for mastication analysis," in System Integration (SII), 2010 IEEE/SICE International Symposium on, dec. 2010, pp. $420-425$.

[8] K. Y. Lim, F. Goh, W. Dong, K. D. Nguyen, I.-M. Chen, S. H. Yeo, H. Duh, and C. G. Kim, "A wearable, self-calibrating, wireless sensor network for body motion processing," in Robotics and Automation, 2008. ICRA 2008. IEEE International Conference on, may 2008, pp. 1017 1022 .

[9] J. Barton, A. Lynch, S. Bellis, B. O’Flynn, F. Murphy, K. Delaney, S. O’Mathuna, P. Repetto, R. Finizio, C. Carvignese, and L. Liotti, "Miniaturised inertial measurement units (IMU) for wireless sensor networks and novel display interfaces," in Electronic Components and Technology Conference, 2005. Proceedings. 55th, 0-0 2005, pp. 1402 -1406 .

[10] "Cross axis effect for AMR magnetic sensors," Honeywell Sensor Products, Tech. Rep. AN215, 2003.

[11] N. El-Sheimy, H. Hou, and X. Niu, "Analysis and modeling of inertial sensors using allan variance," Instrumentation and Measurement, IEEE Transactions on, vol. 57, no. 1, pp. $140-149$, jan. 2008. 